\section{Journal impact factors}

SIR - A correspondent earlier this year (Nature 374, 492; 1995) asserts that: “... Computerized analyses of scientific publications allow immediate comparison of applicants by objective parameters". Following the recent public competitions for full-time university academic positions (concorsi), a group of unsuccessful candidates has levelled accusations at members of the examining commission and demanded that objective approaches, notably the impact factor (IF) and citation index (CI), be adopted in the evaluation of scientific publications of applicants.

The function of the IF is to ascertain the importance of scientific journals and, indirectly, the articles published in them, on the basis of the number of citations they yielded in a period of two years. Applying an IF parameter intended for publications to the university concorso context is, in my view, arbitrary. Some of the protesters have quoted global IFs but have not always explained how they are calculated. One procedure is to divide the IF for each article by the number of authors, but it is far from clear whether such a procedure can be correct. Why stop with two years' citations, when figures for the whole of a candidate's career should be available? What has been the candidate's specific contribution to the research described? It is also crucial to assess the relevance of published research to the discipline of the concorso: a biologist whose published article in Nature obtains a high

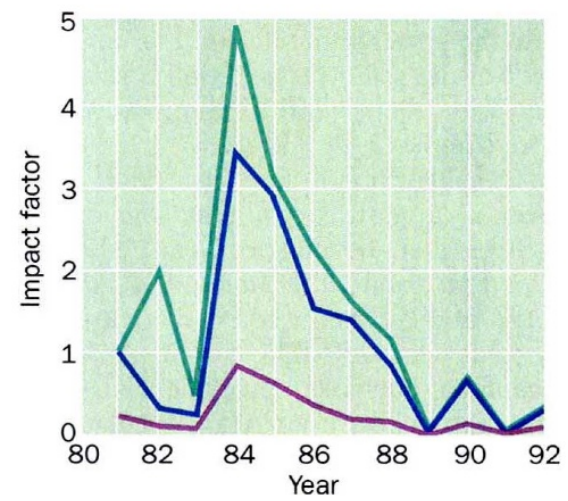

Green: IF Candidate + co-author (1992 value): sum 17.757. Blue: IF candidate + co-author (annual value): sum 12.555. Purple: IF candidate alone (annual value): sum 2.962.

IF should not as a matter of course win the competition for the chair of urology or otorhinolaryngology.

The graph shows the case of a failed candidate who protested because he claimed his IF was high (about 17,000). That figure was arrived at by obtaining a global value of his career (1981-91), by applying to his articles the IF value that the journals in which they appeared had themselves received in 1992 and by neglecting to divide the IF value of each article by the number of co-authors.

In view of these objections, the global IF of the dissatisfied applicant drops from 17.76 to 2.97 . It is also evident from the graph that the candidate had not carried out any valid scientific activity for the five years preceding the concorso. In reality, his publications treat a limited area of the discipline in the concorso, which raises some concern about the applicant's knowledge of the whole subject and his ability to teach it.

To entrust the assessment of university concorso applicants to a computer rather than a committee is, to my mind, misleading and simplistic. To ignore an applicant's knowledge of and competence in the many subdivisions of his discipline, to overlook his career path, particularly in the few years before the competition, and to dismiss the interest expressed in his monographs and works published in national and European journals where the IF is inadequately assessed is damaging to say the least.

\section{Giovanni Motta}

Institute of Otorhinolaryngology,

Faculty of Medicine,

University 'Federico II',

Via Pansini 5,

80131 Naples,

Italy

SIR - Journal impact factors (IFs) are increasingly being used as 'objective' quantitative measures of the quality of output of individuals, research groups or universities, excellence being equated with the ability to publish in journals with high IFs ${ }^{1}$. They are calculated by dividing the number of all citations of articles published in a particular journal during the previous two years by the number of articles published in that journal in those two years ${ }^{2}$.

However, there is a tenfold difference in the IF of the leading journals in different specialist fields, even within the same broad subject. Why is this? I have recently conducted a simple analysis which shows that most of this variation in journal IF is totally independent of the quality of the science. I took a random sample of 28 'good' biological and biomedical journals (defined as those in the top $15 \%$ in their specialist field in the IF league tables ${ }^{2}$, excluding general science and review journals). From the first 5 normal research papers in the most recent issue of each I then calculated the mean time from submission to publication ( = publication lag; range $=4.4-21.0$ months) and the percentage of cited references published within the past two years (=turnover, a measure of how much the discipline relies on recently published material; range $=$ $0-39 \%$ ). Turnover is similar in concept to the 'cited half-life' listed (but strangely never used) in IF tables, but is independent of journal quality. IFs were significantly related to both of my parameters (correlation of In(IF) with publication lag: $r=-0.698, P<0.0001$; with angular transformed turnover: $r=0.834, P<0.0001$; see figure). Both were significant in a stepwise multiple regression predicting IF, indicating that their effects were partially additive (combined $r^{2}=0.717, \mathrm{P}<0.0001$ ). Thus almost $72 \%$ of the tenfold variation in IF between leading journals in different specialist areas was explained by two variables that were entirely unrelated to the scientific quality of either the papers examined or the journals!

Turnover and publication lags tend to be broadly similar within, but not between, specialist fields. Given that IFs

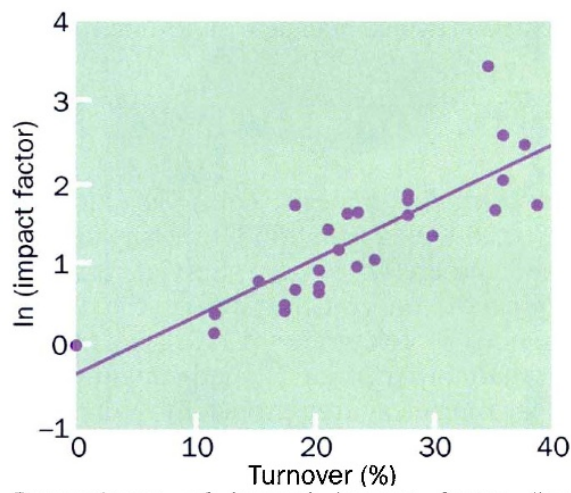

Dependence of journal impact factor (log scale) on turnover of the scientific discipline (angular transformed \% of citations within the past 2 years) - see text for details.

are based entirely on citations appearing within two years of a paper being published, the far higher IF of top biochemical and molecular journals compared to (say) ecological ones is therefore largely an artefact, due to (1) publication lags being far shorter (mean of $6.2 \pm 0.5$ months, c.f. $16.3 \pm 1.2$ ) and (2) a far greater percentage of their cited references being from the past 2 years (mean of $28.2 \pm 2.5 \%$, c.f. $9.5 \pm 1.2 \%$ ). While IFs can be used as measures of journal quality within narrowly defined disciplines, they are next to useless in broader-scale comparisons (for example comparing different biological specialisms) unless these systematic biases are corrected. We should ensure that funding bodies and others who use IFs are aware of the unscientific nature of their current measure of scientific excellence

\section{Neil B. Metcalfe}

Division of Environmental

\& Evolutionary Biology,

Graham Kerr Building, IBLS,

University of Glasgow,

Glasgow G12 8QQ, UK

\footnotetext{
1. Statzner, B., Resh, V.H. \& Kobzina, N.G. Trends Ecol. Evol. 10, 220 (1995)

2. Institute for Scientific Information (ISI) 1992 Journal Citation Reports (ISI, Philadelphia, USA, 1993).
} 